\title{
Overexpression of the regulator of G-protein signaling 5 reduces the survival rate and enhances the radiation response of human lung cancer cells
}

\author{
ZUMIN XU ${ }^{1,3^{*}}$, YUFANG ZUO $^{3 *}$, JIN WANG $^{2 *}$, ZHONGHUA YU ${ }^{3 *}$, FANG PENG $^{1}$, YUANYUAN CHEN $^{2}$,

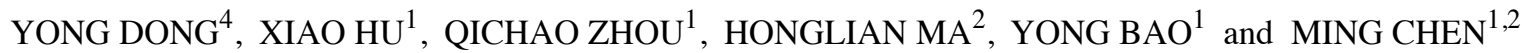 \\ ${ }^{1}$ Department of Radiation Oncology, Sun Yat-Sen University Cancer Center, State Key Laboratory of Oncology in South China, \\ Collaborative Innovation Center for Cancer Medicine, Guangzhou, Guangdong 510060; ${ }^{2}$ Zhejiang Cancer Hospital, \\ Hangzhou, Zhejiang 310012; ${ }^{3}$ Cancer Center, Affiliated Hospital of Guangdong Medical College, Zhanjiang, \\ Guangdong 524000; ${ }^{4}$ Cancer Center, Shilong People's Hospital, Dongguan City, Guangdong 523321, P.R. China
}

Received November 27, 2014; Accepted January 13, 2015

DOI: $10.3892 / o r .2015 .3917$

\begin{abstract}
Regulator of G protein signaling 5 (RGS5) belongs to the R4 subfamily of RGS proteins, a family of GTPase activating proteins, which is dynamically regulated in various biological processes including blood pressure regulation, smooth muscle cell pathology, fat metabolism and tumor angiogenesis. Low-expression of RGS5 was reported to be associated with tumor progression in lung cancer. In the present study, we examined the potential roles of RGS5 in human lung cancer cells by overexpressing RGS5 in the cancer cells and further explored the underlying molecular mechanisms. The RGS5 gene was cloned and transfected into the human lung cancer cell lines A549 and Calu-3. The cells were tested for apoptosis with flow cytometry, for viability with MTT, for mobility and adhesion capacity. The radiosensitization effect of RGS5 was measured by a colony formation assay. The mechanisms of RGS5 functioning was also investigated by detection of protein expression with western blot analysis, including PARP, caspase 3 and 9, bax, bcl2, Rock1, Rock2, CDC42, phospho-p53 (Serine 15) and p53. The present study demonstrated that RGS5 overexpression remarkably induced apoptosis in human lung cancer cells, which was suggested to be through mitochondrial mechanisms. Overexpression of RGS5 resulted in significantly lower adhesion and migration abilities of the lung cancer cells $(\mathrm{P}<0.01)$. Furthermore, overexpression of RGS5 sensitized the lung cancer cells to
\end{abstract}

Correspondence to: Dr Ming Chen or Dr Yong Bao, Sun Yat-sen University Cancer Center, 651 East Dongfeng Road, Guangzhou, Guangdong 510060, P.R. China

E-mail: chenming@sysucc.org.cn

E-mail: baoyong@sysucc.org.cn

*Contributed equally

Key words: regulator of G-protein signaling 5, lung cancer, radiation, radiosensitization radiation. In conclusion, the present study showed that RGS5 played an inhibitory role in human lung cancer cells through induction of apoptosis. Furthermore, RGS5 enhanced the cytotoxic effect of radiation in the human lung cancer cells. Our results indicated that RGS5 may be a potential target for cancer therapy.

\section{Introduction}

Regulator of $\mathrm{G}$ protein signaling (RGS) proteins is a family of GTPase activating proteins, which interact directly with $\alpha$ subunit of heterotrimeric $\mathrm{G}$ proteins to accelerate the GTP hydrolysis activity of the $\alpha$ subunit and negatively regulate the $\mathrm{G}$ protein signaling $(1,2)$. All the RGS family members contain an essential 120-amino acid RGS domain. Regulator of $\mathrm{G}$ protein signaling 5 (RGS5) belongs to the R4 subfamily of RGS proteins, which consists mostly of small RGS proteins containing only short sequences beyond the RGS domain (3). RGS5 expression is abundant in pericytes and vascular smooth muscle cells $(4,5)$ and high levels of RGS5 have been detected in the heart, lung, skeletal muscle and small intestine, while lower levels have been found in the brain, placenta, liver, colon and leukocytes (6). RGS5 has been implicated in many pathophysiological processes including cardiac hypertrophy (7), blood pressure regulation (8), atherosclerosis $(9,10)$, lipid metabolism (11), bronchial smooth muscle cell contraction and asthma (12). RGS5 was markedly downregulated during the endothelial cell morphogenesis in an in vitro human capillary tube formation model (13). RGS5 levels were elevated in pericytes during wound healing and ovulation, suggesting a strong correlation between the RGS5 expression and the active vessel remodeling $(14,15)$.

Substantial evidence indicates an active role of RGS5 in tumor angiogenesis. RGS5 is found highly expressed in the vasculature of several tumors. RGS5 mRNA is expressed in the endothelial cells of tumor vessels, but not in the endothelial cells of the normal tissues in renal cell carcinoma (RCC) (16), hepatic cell carcinoma (HCC) (17) and ovarian carcinoma (18). RGS5 mRNA is specifically upregulated in 
the vasculature of premalignant lesions during the 'angiogenic switch' and further elevated in tumor vessels in a mouse model of pancreatic islet cell carcinogenesis (14). Elevated expression of RGS5 has been reported in tumor cells in gastric tumor (19), parathyroid (20) and non-small cell lung cancer (NSCLC) (21). Yet, the fundamental role RGS5 played in the neoplasm and its mechanisms are still to be clarified.

In the present study, we investigated the potential role of the high levels of RGS5 in human lung cancer cells and further explored the underlying molecular mechanisms.

\section{Materials and methods}

Ethics statement. The present study conforms to the principles outlined in the Declaration of Helsinki, and was approved by the Medical Ethics Committee of Sun Yat-Sen University. Written informed consents were obtained from the donors.

Cells and cultures. Human lung cancer cell lines A549, Calu-3, H1299 and SK-MES-1 were obtained from the Cell Bank of the Chinese Academy of Sciences (Shanghai, China), and cultured in RPMI-1640 medium supplemented with $10 \%$ (v/v) fetal bovine serum (FBS) (both from Life Technologies, Grand Island, NY, USA) at $37^{\circ} \mathrm{C}, 5 \% \mathrm{CO}_{2}$ in a humidified incubator. Human umbilical vein endothelial cells (HUVECs) were freshly isolated from the human umbilical cord veins, and incubated in the human endothelial-SFM basal growth medium supplemented with $20 \%$ fetal calf serum, $100 \mu \mathrm{g} / \mathrm{ml}$ streptomycin, $100 \mathrm{U} / \mathrm{ml}$ penicillin, $5 \mu \mathrm{g} / \mathrm{ml}$ amphotericin B (Life Technologies), $2 \mathrm{mmol} / 1$ l-glutamine, $15 \mathrm{mg} / 1 \mathrm{ECGS}$ (Upstate Biotechnology, Lake Placid, NY, USA) (22).

mRNA extraction and reverse transcription PCR. mRNA extractions were performed with an ultrapure RNA purification kit (Kangweishiji Biotech Co., Ltd., China) according to the manufacturer instructions. Approximately 500 ng of RNA was reverse transcribed into complementary DNA using a PrimeScript RT Reagent kit (Takara, Shiga, Japan). Relative quantity of RGS5 mRNA was determined using GAPDH as an internal control. PCR was performed with the primers for RGS5, 5'-atgtgcaaaggacttgcagc-3'/5'-ctacttgattaactcctgat-3'; and GAPDH, 5'-tccaccaccetgttgctgta-3'/5'-accacagtccatgc atcac-3'.

Cloning of RGS5 coding region and cell transfection. The complete RGS5 coding region was cloned into the pTRiEX Neo expression vector (Novagen, USA) for transient transfection of cancer cell lines. The RGS5 coding region was amplified from cDNA of HUVECs using primers 5'-cgcggatccgggatcc gatgtgcaaaggacttgcagc-3' and 5'-ccgctcgagcttgattaactcetgataaa ac-3' which contain restriction site tags for BamHI and XhoI, respectively. The RGS5 coding region was then cloned into the pTRiEX Neo vector (Clontech, USA). Human lung cancer cell lines A549 and Calu-3 were transiently transfected with pTRiEX vectors or pTRiEX-RGS5 plasmids using Attractene Transfection Reagent (Qiagen, Germany) according to the protocol of the manufacturer.

Cell viability. Cell viability was measured by MTT assays as previously described (23). At various time-points following transfection, MTT solution was added at a final concentration of $0.5 \mathrm{mg} / \mathrm{ml}$ and the cells were incubated for $4 \mathrm{~h}$ at $37^{\circ} \mathrm{C}$. Formazan crystals in the viable cells were solubilized with dimethyl sulfoxide and the absorbance was measured at $570 \mathrm{~nm}$.

Colony formation assay. The cells were seeded into 6-well plates at a density of 500 cells/well. For radiation groups, 6 -well plates were irradiated with 2 or 6 Gy X-ray $12 \mathrm{~h}$ after seeding. Irradiation was performed at room temperature using RS 2000 X-ray Biological Irradiator (Rad Source Technologies, USA) at a dose rate of $1 \mathrm{~Gy} / \mathrm{min}$ through a $0.2-\mathrm{mm}$ copper filter. The plates were incubated for 10 days at $37^{\circ} \mathrm{C}$ with the growth medium being replaced every 3 days. The colonies were fixed with methanol and stained with $0.5 \%$ crystal violet (in methanol:water, 1:1). The number of the colonies containing at least 50 cells was then counted. Data are presented as means $\pm \mathrm{SE}(\mathrm{SEM})$ of at least three independent experiments.

Apoptosis assay. The cells were collected at $36 \mathrm{~h}$ after transfection and the apoptotic cells were analyzed using flow cytometry (FACS) with an Annexin V/propidium iodide (PI)-staining assay according to the instructions of the manufacturer (Kaiji Biotechnology Co., Nanjing, China).

Cell adhesion assay. Ninety-six-well plates were coated with human fibronectin (BD Biosciences) at a final concentration of $2 \mu \mathrm{g} / \mathrm{ml}$ overnight at $4^{\circ} \mathrm{C}$. The plates were washed with $1 \%$ bovine serum albumin in phosphate-buffered saline (PBS) to block non-specific cell adhesion. Twelve hours after being transfected with either pTRiEX or pTRiEX-RGS5 plasmids, the cells were trypsinized and seeded at $5 \times 10^{4}$ cells/well, and then incubated for $1 \mathrm{~h}$. The plates were washed with PBS to remove non-adherent cells, fixed with formalin and followed by staining with crystal violet for $15 \mathrm{~min}$ and solubilized with $1 \%$ SDS. The absorbance was measured at $570 \mathrm{~nm}$ by a microplate reader (Tecan Sunrise, Germany). All the experiments were performed in triplicates and repeated at least three times.

Cell migration assay. The in vitro migration ability of the cells was measured using an $8-\mu \mathrm{m}$ Boyden chamber assay (Corning, USA). Twelve hours after transfection with pTRiEX or pTRiEX-RGS5 plasmids, the cells were trypsinized and seeded into the upper chamber. A total of $2 \times 10^{4}$ cells in $0.2 \mathrm{ml}$ of $2 \%$ FBS medium was placed in the upper chamber, whereas the lower chamber was loaded with $0.8 \mathrm{ml}$ medium containing $20 \% \mathrm{FBS}$. After incubation at $37^{\circ} \mathrm{C}$ for $12 \mathrm{~h}$, non-migratory cells were wiped off with PBS-rinsed cotton swabs. The filters were then fixed in ethanol, stained with crystal violet. The stained cells were counted under a microscope (Olympus, Tokyo, Japan). Values for migration were expressed as the average number of migrated cells/microscopic field over six fields/assay from three independent experiments.

Western blot analysis. The cells were lysed in lysis buffer [20 mM Tris ( $\mathrm{pH}$ 7.4), $250 \mathrm{mM} \mathrm{NaCl}, 2 \mathrm{mM}$ EDTA, 0.1\% Triton X-100, $0.4 \mathrm{mM} \mathrm{PMSF}]$ and heated at $100^{\circ} \mathrm{C}$ for $10 \mathrm{~min}$. The protein concentration was determined using the Bio-Rad Protein Assay kit (Bio-Rad, Hercules, CA, USA). The samples 

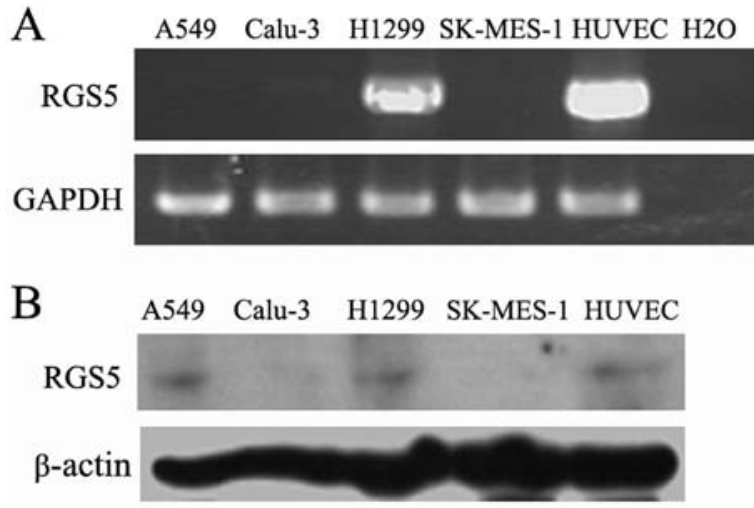

Figure 1. Expression of RGS5 in human non-small cell lung cancer cell lines. (A) RT-PCR analysis of RGS5 mRNA levels, (B) western blot analysis of RGS5 protein levels in cancer cell lines. Human umbilical vascular endothelial cells (HUVECs) were used as a positive control. RGS5, regulator of $\mathrm{G}$ protein signaling 5 .

were separated by SDS-PAGE and transferred to the PVDF membranes (Bio-Rad). The membranes were blocked in 5\% milk for $1 \mathrm{~h}$. Protein expression was detected using primary antibodies incubated overnight at $4^{\circ} \mathrm{C}$. The membranes were washed and incubated for $1 \mathrm{~h}$ with HRP-conjugated secondary antibodies at room temperature. The following primary antibodies were used: anti-RGS5 $(1: 1,000)$, anti-PARP $(1: 1,000)$, anti-caspase $3(1: 1,000)$, anti-caspase $9(1: 1,000)$, anti-bax (1:2,000), anti-bcl (1:1,000), anti-Rock1 (1:1,000), anti-Rock2 (1:1,000), anti-CDC42 (1:1,000), anti-Phosphop53 (Serine 15) (1:1,000) and anti-p53 (1:1,000). Horseradish peroxidase-coupled anti-rabbit or anti-mouse antibodies (Vector Laboratories) were used at a dilution of 1:1,000. The blots were probed with an anti- $\beta$-actin antibody (Sigma) for equal loading.

Statistical analysis. Data are presented as mean \pm SEM. Differences of the variables between the groups were analyzed by one-way ANOVA test. Differences were considered significant at $\mathrm{P}<0.05$.

\section{Results}

Expression of RGS5 in human lung cancer cells. Four human non-small cell lung cancer cell lines were tested for the expression levels of RGS5 using RT-PCR and western blot analysis with HUVECs as positive controls. RGS5 was differentially expressed in human lung cancer cells, with high levels in H1299 cells and undetectable levels in A549, Calu-3 and SK-MES-1 cells (Fig. 1).

Overexpressing of RGS5 in A549 and Calu-3 cells inhibits growth and clongenic survival. As shown in Fig. 2, A549 and Calu-3 cells that were transfected with RGS5-expressing plasmid had markedly increased levels of RGS5 mRNA and protein, compared to the cells transfected with the vector control.

Upon transfection with RGS5-expressing plasmid, both A549 and Calu-3 cells showed reduced cell density at $36 \mathrm{~h}$ post-transfection, compared to the cells receiving vector control or no plasmid (Fig. 3A). An MTT assay was employed to study the effects of overexpression of RGS5 on the cell growth. As shown in Fig. 3B, expression of RGS5 was able to significantly inhibit the growth of both A549 and Calu-3 cells. Compared to the cells receiving vector control, growth inhibition of A549 and Calu- 3 cells was 44.4 and $39.27 \%$ at $48 \mathrm{~h}$, and $54.3,44.7 \%$ at $72 \mathrm{~h}$, respectively (Fig. 3C). The in vitro clonogenic assay further confirmed that overexpression of RGS5 asserted an inhibitory effect on the cell proliferation. Compared to the non-transfected control or the vector transfected cells, overexpression of RGS5 inhibited colony formation of both A549 and Calu-3 cells by 46.8 and $52.5 \%$, respectively (Fig. 3D).

RGS5 induces lung cancer cell apoptosis through activation of the mitochondrial pathway. To explore the possible mechanism responsible for RGS5-mediated cell growth inhibition, cellular apoptosis was evaluated by Annexin V-PI staining and analyzed by flow cytometry. Both A549 and Calu-3 cells have increased populations undergoing apoptosis when transfected with RGS5-expressing plasmid (Fig. 4A and B). The proportion of the apoptotic cells untreated, transfected with pTRiEX
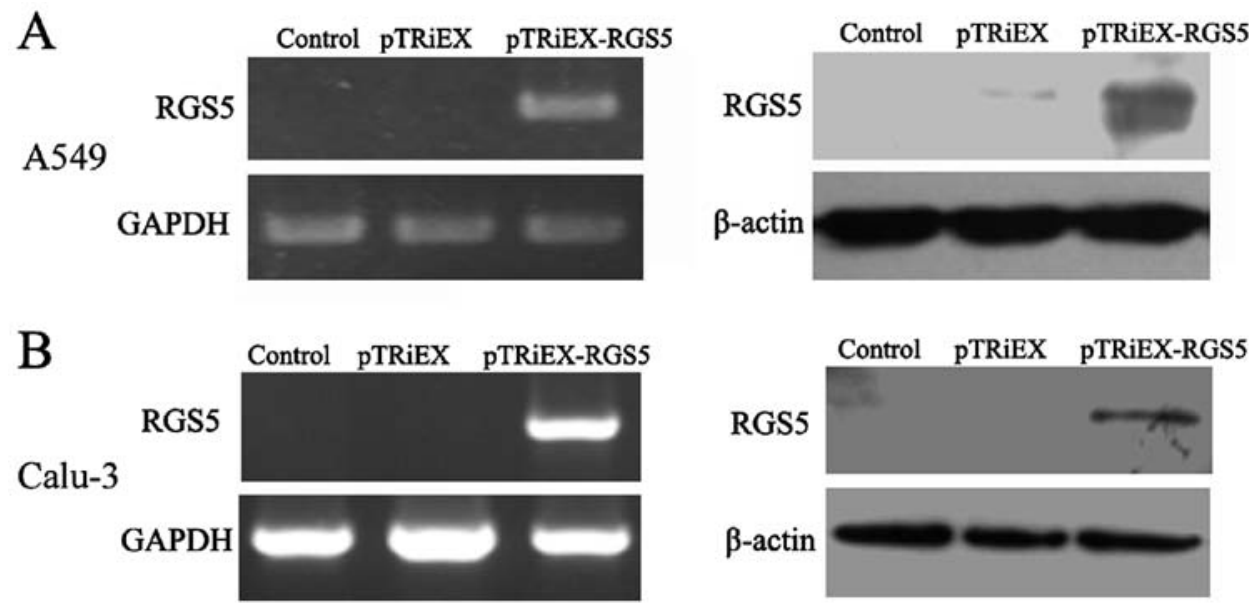

Figure 2. Analysis of expression of RGS5 after transfection of RGS5-expressing plasmid into (A) A549 and (B) Calu-3 cells via RT-PCR and western blot analysis. RGS5 protein expression was detected in the control (non-transfected cells), the vector (cells transfected with vector only) and RGS5 (cells transfected with pTRiEX-RGS5 plasmids). RGS5, regulator of G protein signaling 5. 
A

A Control

pTRiEX

pTRiEX-RGS5

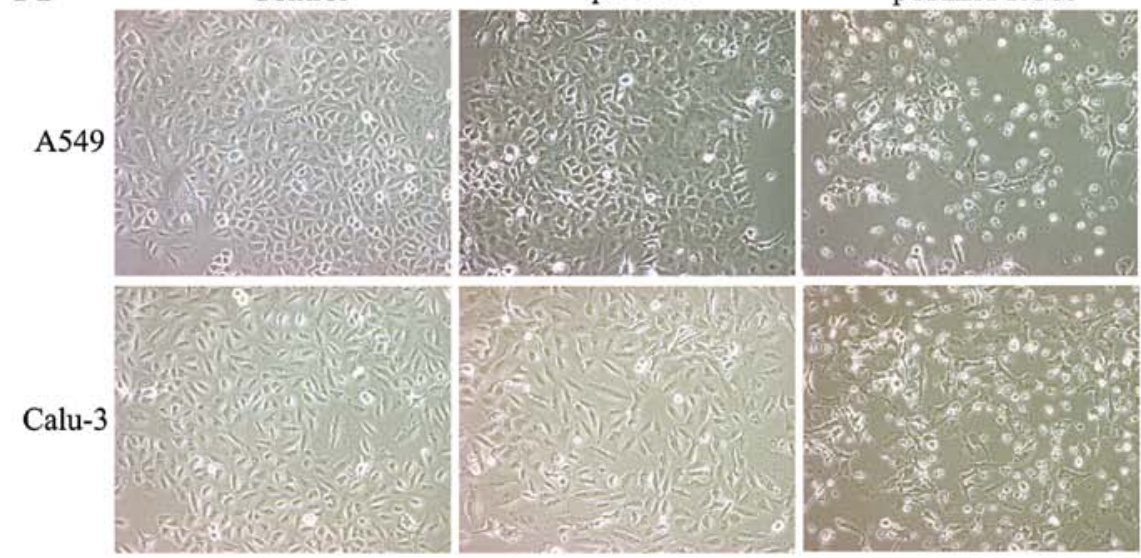

B

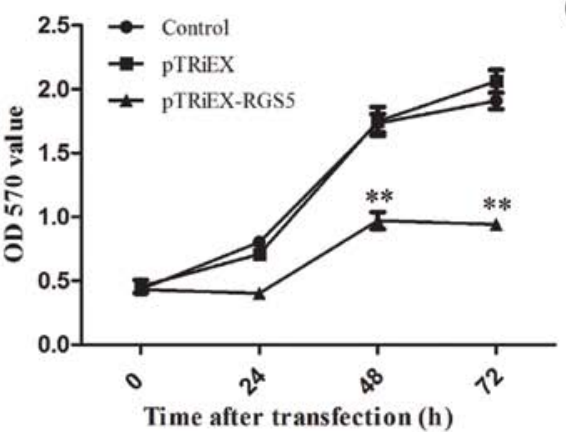

C

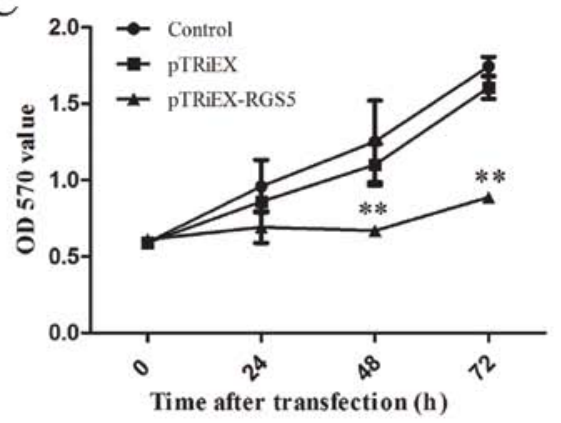

$\mathrm{D}$

D Control

pTRiEX

pTRiEX-RGS5

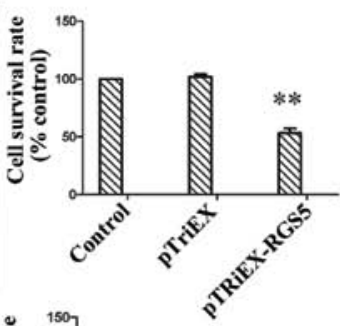

A549
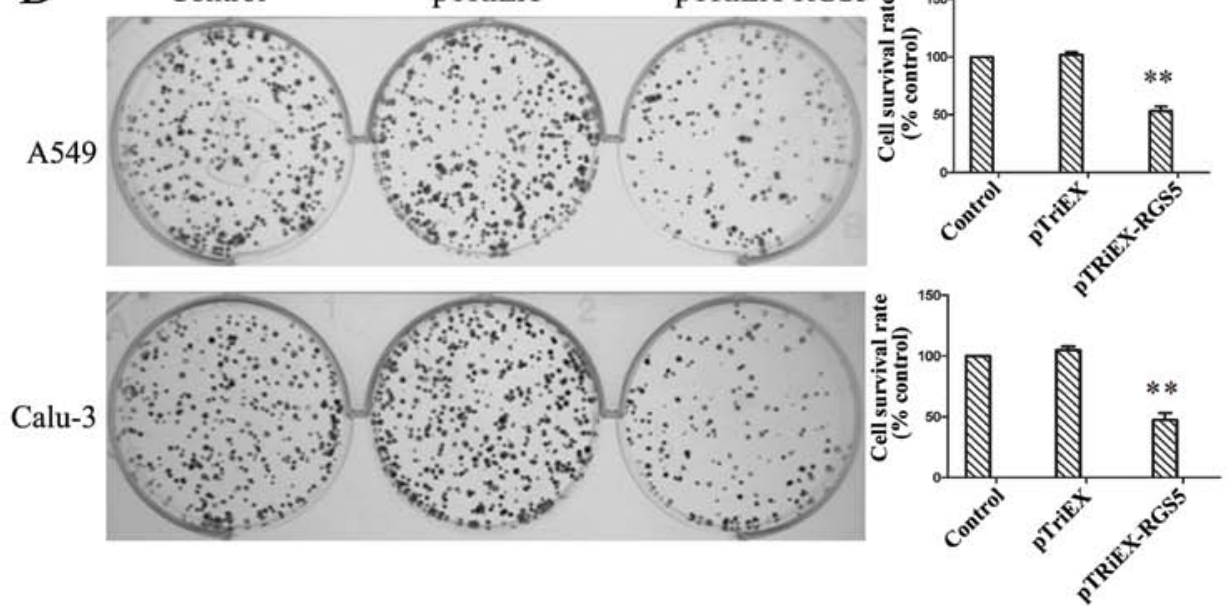

Figure 3. Cell survival and colony formation in A549 and Calu-3 cells transfected with RGS5. (A) Representative images on morphology of cells $36 \mathrm{~h}$ with/without transfection; cell viability of (B) A549 and (C) Calu-3 cells at various time-points following RGS5 transfection measured by MTT assays; (D) influence of RGS5 on the number of colony-forming cells as evaluated by a clonogenic assay. The statistical data shown are the means from three independent experiments. Each value is the mean \pm SEM. ${ }^{* *} \mathrm{P}<0.01$, compared to the control group as analyzed by one-way ANOVA test. RGS5, regulator of G protein signaling 5 .

or pTRiEX-RGS5 at $36 \mathrm{~h}$ post-transfection in A549 cells was $1.3 \pm 0.2,3.4 \pm 0.6$ and $19.6 \pm 2.3 \%$, respectively; whereas $3.2 \pm 0.8$, $3.0 \pm 0.9$ and $12.8 \pm 1.8 \%$, respectively, in Calu- 3 cells.

To determine whether caspases were involved in the RGS5-induced apoptosis, we studied the activation of caspase 3 and 9 and the cleavage of PARP. As shown in Fig. 4C, A549 and Calu-3 cells overexpressing RGS5 had decreased levels of pro-caspase 3 and 9, whereas the levels of cleaved form of PARP in the same cell lines were increased, indicating the increased activation of both caspases.
Mitochondria play a critical role in regulating apoptosis. Pro-apoptotic proteins Bax and Bak, as well as anti-apoptotic protein $\mathrm{Bcl}-2$ are involved in regulating the release of mitochondrial protein cytochrome $c$ to initiate the apoptotic cascade. We investigated the effect of RGS5 expression on the levels of Bax, Bak and Bcl-2 proteins. As shown in Fig. 4D, cells transfected with pTRiEX-RGS5 had increased levels of Bax and Bak protein but decreased levels of Bcl-2. Therefore, the ratio of pro-apoptotic/anti-apoptotic was increased when RGS5 was overexpressed in the cells. These results indicated 
A



B



Annexin V
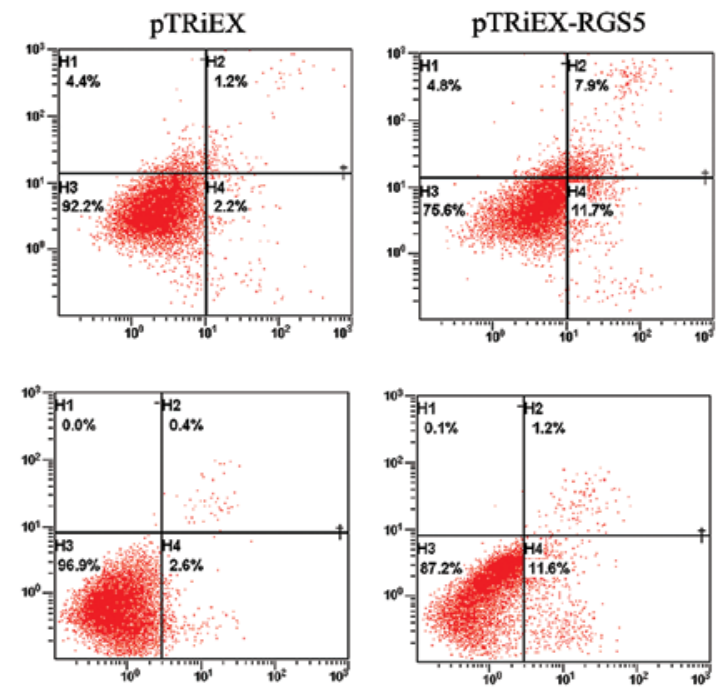

D

A549
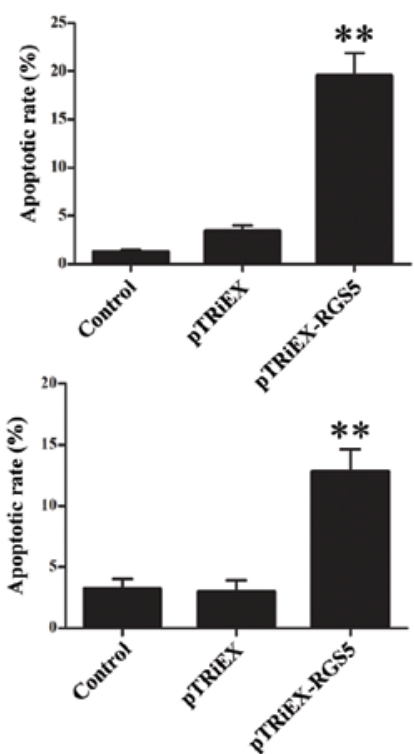

Calu-3

pTRIEX pTRIEX-RGSS
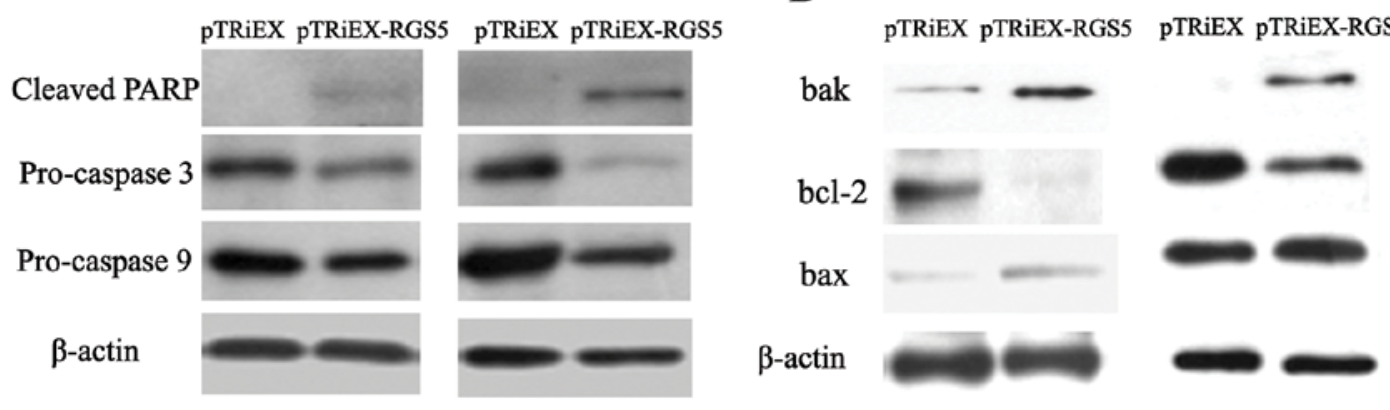

Figure 4. Apoptosis in human lung cancer cell lines that overexpress RGS5. The effects of RGS5 on the apoptosis of (A) A549 and (B) Calu-3 were studied with Annexin V and PI staining followed by FACS analysis. ${ }^{* *} \mathrm{P}<0.01$, compared to the control group as analyzed by the one-way ANOVA test. (C) Western blot analysis on the expression of caspase 3 and 9 pro-enzymes and cleaved PARP in A549 and Calu-3 cells transfected with pTRiEX vector or pTRiEX-RGS5. (D) Western blot analysis on the expression of bak, bcl-2 and bax in A549 and Calu-3 cells transfected with pTRiEX vector or pTRiEX-RGS5. RGS5, regulator of $\mathrm{G}$ protein signaling 5 .

that RGS5 induced apoptosis via mitochondrial pathway in human lung cancer cells.

RGS5 inhibits adhesion and migration of lung cancer cells. Previous studies have reported that the low level of RGS5 is associated with an increased incidence of lymph node metastasis and more invasion in non-small cell lung cancer patients (21). Thus, to understand whether RGS5 is responsible for the observed phenotype, we investigated whether the increased levels of RGS5 in human lung cancer cells would inhibit cell adhesion or migration.

Cell adhesion was assessed on the fibronectin-coated surface. A549 and Calu-3 cells were transfected with RGS5 expressing plasmid or vector only control, and were allowed to adhere to the fibronectin-coated surface for $1 \mathrm{~h}$. As shown in Fig. 5A, the overexpression of RGS5 reduced A549 and Calu-3 cell adhesion to fibronectin-coated surfaces by 45.9 and $28.9 \%$ respectively, compared to the control cells. The differences were apparent from the microscope images.

Similarly, the effect of RGS5 on cell migration was assessed in the RGS5 expressing A549 and Calu-3 cells. The cells were seeded in the upper chamber, and allowed to migrate through an $8-\mu \mathrm{m}$ filter. Over a $12-\mathrm{h}$ incubation period, the number of cells penetrated through the filter in vector- and the RGS5transfected A549 and Calu-3 cells was 39.9 $\pm 11.2,10.23 \pm 4.42$, $44.56 \pm 10.6$ and $13.08 \pm 3.37 /$ field, respectively (Fig. 5B). To determine the mechanisms of the observation, expression levels of several cell migration markers including Rock1, Rock2, cdc42 and MMP-2 were studied in these cells by western blot analysis. As demonstrated in Fig. 5C, in A549 or Calu-3 cells transfected with pTRiEX-RGS5, the levels of all four proteins were decreased compared to the cells transfected with pTRiEX.

RGS5 enhances the cytotoxic effects of X-ray irradiation on lung cancer cells. To determine whether increased levels of RGS5 increased the sensitivity of lung cancer cells to radiation, we performed a colony-forming assay with cells expressing higher levels of RGS5 followed by irradiation treatment. The cells transfected with either pTRiEX-RGS5 or pTRiEX were subjected to radiation exposure ( 2 Gy). Radiation of the cells expressing RGS5 resulted in further inhibition of colony formation (Fig. 6A and B). These results suggested that RGS5 enhances the cytotoxic effect of radiation treatment.

To further explore the mechanism of colony formation inhibition after transfection with RGS5 and radiation, the two cell 
A

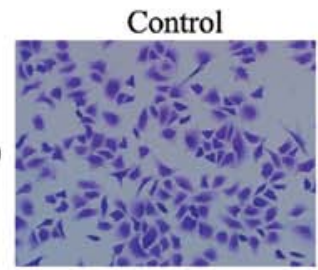

A549

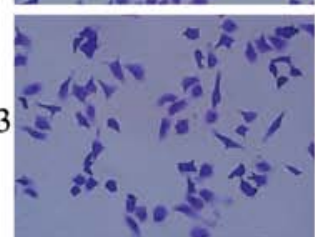

Calu-3

B

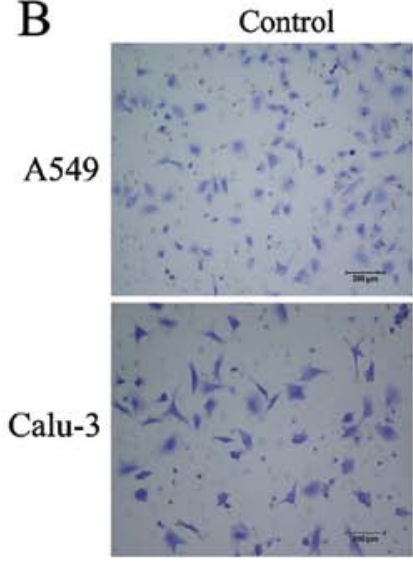

$\mathrm{C}$



pTRiEX
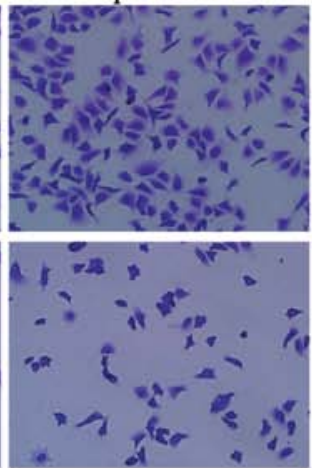

pTRiEX


Calu-3
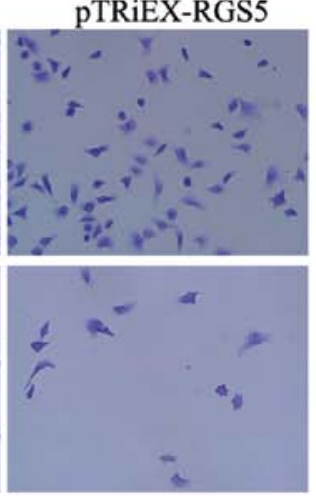

pTRiEX-RGS5

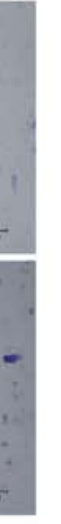

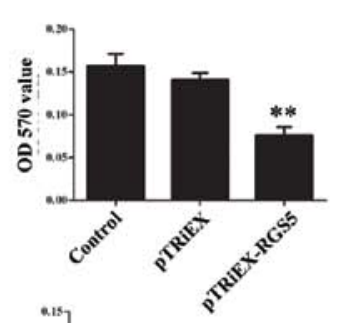
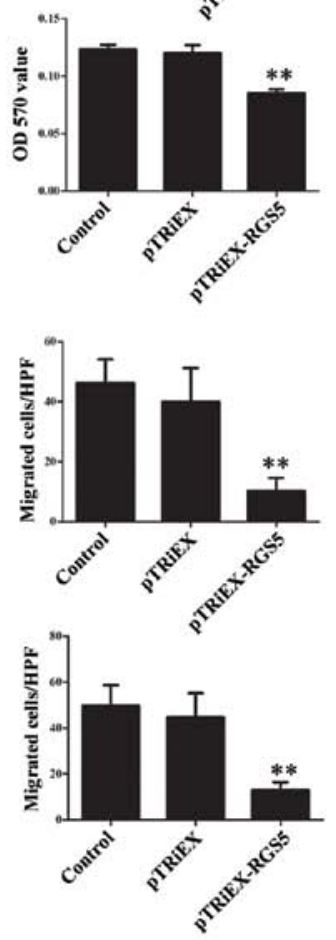

Figure 5. Adhesion and migration of human lung cancer cell lines A549 and Calu-3 overexpressing RGS5. (A) Effects of RGS5 overexpression on cell adhesion in cancer cells. The adherent cells in each well were observed microscopically and representative images are shown. All absorbance values were measured using a photometer at $570 \mathrm{~nm}$ for crystal violet. (B) Effects of RGS5 overexpression on cell migration in cancer cells. Representative microscopic images are shown. Data are mean \pm SD from three independent determinations. ${ }^{* *} \mathrm{P}<0.01$ when compared with the parental and the vector-transfected cells by one-way ANOVA. RGS5, regulator of G protein signaling 5.

lines were transfected with pTRiEX-RGS5, irradiated at $6 \mathrm{~Gy}$, and then the cell lysates were analyzed by western blot analysis. Combined radiation treatment with RGS5 overexpression led to increased levels of cleaved PARP, cleaved caspase 3 and 9 in both cell types (Fig. 6C). It is known that radiation treatment would lead to the activation of p53 pathway (24). We found that phosphorylation of p53 at S15 was increased upon radiation, and was further increased when RGS5 was overexpressed in the radiated cells (Fig. 6C). As a result, the p53 protein levels were increased in the radiated cells.

\section{Discussion}

G-protein-coupled receptors (GPCRs) are a large family of versatile membrane proteins involved in a wide range of physiological processes and pathological diseases (25). GPCRs classically transmit their signal via the activation of $\mathrm{G}$ protein heterotrimer, which exchanges GDP for GTP, dissociates into two parts and is released from the receptors. Both free GTP-bound $\alpha$ subunit and $\beta \gamma$ dimers are capable of activating downstream effectors. Signaling is terminated 
A

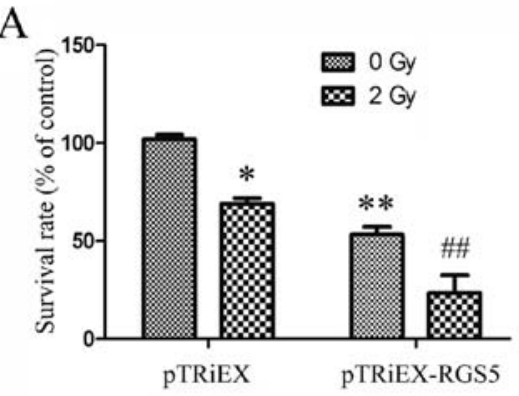

B

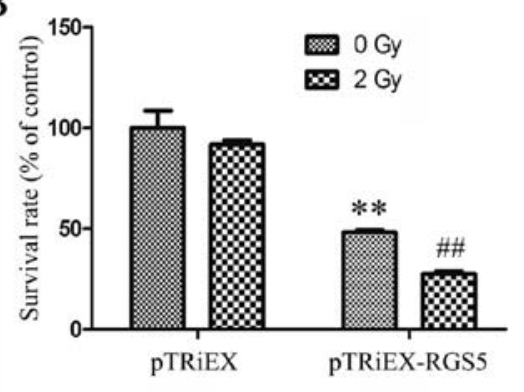

$\mathrm{C}$

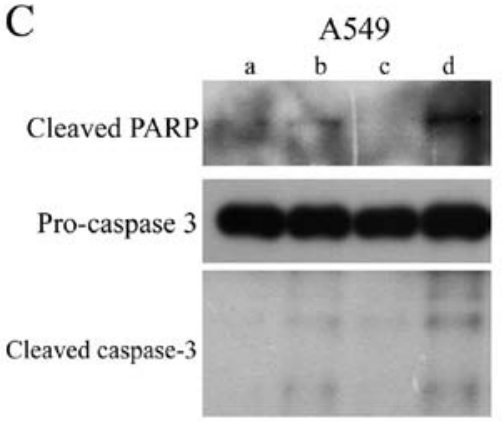

Pro-caspase 9

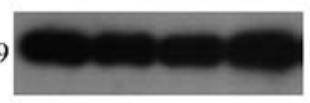

Cleaved-caspase

$\beta$-actin


p-p53 (Ser 15)

p53

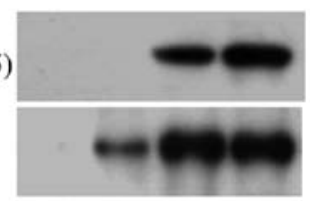

$\beta$-actin

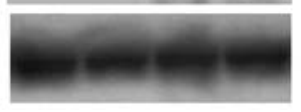

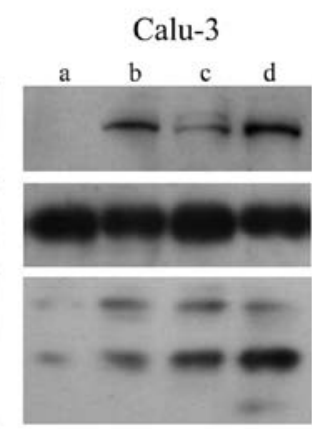


Figure 6. Radio-sensitization effect of RGS5. Clonogenic assay of human lung cancer cells after RGS5 transfection followed by radiation was performed. (A) A549 and (B) Calu-3 were transfected with pTRiEX or pTRiEX-RGS5 plasmid alone or combined with 2 Gy of radiation $24 \mathrm{~h}$ after transfection. The colonies were counted on day 10 . The data were representative of three independent experiments. ${ }^{*} \mathrm{P}<0.05$ compared to pTRiEX-transfected group, ${ }^{* *} \mathrm{P}<0.01$, compared with the control group; ${ }^{\# \#} \mathrm{P}<0.01$, compared with the pTRiEX-RGS5-transfected group. (C) Western blot analysis of cleaved PARP and p53 levels in A549 and Calu-3 cells transfected with pTRiEXor pTRiEX-RGS5 plasmid, with or without 6 Gy of radiation treatment $12 \mathrm{~h}$ after transfection. The whole cell lysate was collected $24 \mathrm{~h}$ after radiation. a, pTRiEX; b, pTRiEX-RGS5; c, pTRiEX + radiation; d, pTRiEX-RGS5 + radiation. RGS5, regulator of G protein signaling 5 .

when $\alpha$ subunit hydrolyzes GTP, returns to the GDP-bound state and re-associates with $\beta \gamma$ dimers to form inactive heterotrimers (26). RGS promotes the hydrolysis of GTP, thus, reduces the life span of the active form of $\alpha$ subunit. RGS proteins are emerging as important negative regulators of G-protein-coupled receptor (GPCR) signaling. Growing evidence has demonstrated that RGS proteins are potential drug targets in pathologies including central nervous system diseases, cardiovascular diseases and diabetes. Targeting RGS proteins with small molecule modulators or inhibitors provide specific control or treatment of pathophysiological conditions $(27,28)$.

Despite the critical roles GPCRs and G proteins play in tumor and metastasis (29), studies on the function of RGS protein in cancer cells are still lacking. However, several studies point to the roles of RGS in regulating cancer cell proliferation. Overexpression of RGS3 in HL-60 promoted cell apoptosis (30). Overexpression of RGS16 inhibited epidermal growth factor (EGF)-induced proliferation and Akt phosphorylation in MCF7 breast cancer cells (31). Altman et al (32) showed that inducible RGS5 expression significantly reduced proliferation of HeyA8-MDR ovarian cancer cells. Maity et al (33) observed that RGS6 induced breast cancer cell apoptosis.

In the present study, it was demonstrated that overexpression of RGS5 significantly reduced the proliferation of human lung cancer cells by inducing apoptosis. Previous studies have reported that RGS5 is upregulated by hypoxia and overexpression of RGS5 induces apoptosis in HUVECs (34). It was demonstrated that in human lung cancer cells, the overexpression of RGS5-induced the expressions of pro-apoptotic proteins Bax and Bak; while it reduced the expression of anti-apoptotic protein $\mathrm{Bcl}-2$. Bax, Bak and Bcl-2 are known to be involved in the regulation of the mitochondrial pathway of apoptosis (35). As a consequence, activation of caspase 3 and 9 was also observed in the RGS5 overexpressing cells.

A previous study indicates an association between the low level of RGS5 and an increased lymph node invasion of the human non-small cell cancer. In addition, RGS4 is found to be involved in reducing breast cancer metastatic abilities in vitro $(36,37)$. We found that when overexpressed, RGS5 decreased the adhesion and migration abilities of human lung cancer cells. Accordingly, several cell migration markers including Rock1, Rock2, cdc42 and MMP-2 were downregulated in RGS5 expressing cells $(38,39)$. Cancer cell migration is believed to be the initial step in metastasis, in which primary tumor cells migrate and invade neighboring tissues and enter the circulation to establish new or secondary tumor sites during metastasis (40). Thus, inhibition of cancer cell migration represents one of the potent strategies for cancer treatment. To our knowledge, this is the first demonstration of the impact of RGS5 overexpression on tumor cell migration. 
Our findings partially explained why lower levels of RGS5 are strongly associated with cancer vascular invasion and lymph node metastasis in NSCLC and indicate RGS5 as a promising target for cancer therapy.

RGS proteins have been shown to modulate the responsiveness of tumor cells to chemotherapeutic agents. The cytotoxic effects of cisplatin, vincristine and docetaxel were reduced following RGS10 and RGS17 knockdown in SKOV-3 as well as MDR-HEYA8 cells (41). RGS6 was shown to mediate the activation of ATM and p53 by doxorubicin, and genetic loss of RGS6 dramatically impaired doxorubicininduced activation of ATM and p53 in breast cancer cells (42). Our observation also pointed to a possible role of RGS5 in increasing the sensitivity of the human lung cancer cells to radiation. Colony formation of both cell lines was reduced when RGS5 was overexpressed in the cells, and it was further reduced when these cells were irradiated. Not surprisingly, p53 pathway was activated upon radiation. It is known that p53 promotes apoptosis through modulating the mitochondrial apoptosis pathway. We observed increased levels of cleaved PARP in the RGS5 expressing cells, and they were further increased when the cells were irradiated. We found that RGS5 overexpression led to an increased level of p53, at least in the A549 cells; whereas the effect was less apparent in Calu-3 cells. This may be the explanation for the changes in the levels of pro-apoptotic and anti-apoptotic Bcl-2 proteins in the RGS5 overexpressing cells. Although the exact underlying mechanism of these observations still requires investigation, to our best knowledge, this is the first study on the antitumor effects of RGS protein combined with radiation.

The present study has noticeable limitation in that expression of the RGS5 was transient, which may bring the question of consistency of the transfection rate and the expression level of each experiment. During the study, each experiment was coupled with detection of the RGS5 expression for validation of the data. Our study used an inducible expression system in cancer to further analyze the function of RGS5.

In conclusion, we demonstrated the effects of RGS5 overexpression in growth inhibition, apoptosis and reduction of cell migration in human lung cancer cells. These findings provided evidence that RGS5 played an inhibitory role in human lung cancer cells, which explained the pathoclinical observation that high expression of RGS5 as a favorable prognostic factor in NSCLC patients. Furthermore, RGS5 was shown to enhance the antitumor effects of radiation in human lung cancer cells. Collectively, our results implied that RGS5 may be a promising target for cancer therapy.

\section{Acknowledgements}

The present study was supported by the National Nature Science Foundation of China (nos. 30872974, 81172116 and 81201736), the Science and Technology Planning Project of Guangdong Province, China (no. 2009B030801154) and the $\mathrm{PhD}$ Programs Foundation of Guangdong Medical College (XB1334).

\section{References}

1. Koelle MR: A new family of G-protein regulators - the RGS proteins. Curr Opin Cell Biol 9: 143-147, 1997.
2. Willars GB: Mammalian RGS proteins: Multifunctional regulators of cellular signalling. Semin Cell Dev Biol 17: 363-376, 2006.

3. Bansal G, Druey KM and Xie Z: R4 RGS proteins: Regulation of G-protein signaling and beyond. Pharmacol Ther 116: 473-495, 2007.

4. Bondjers C, Kalén M, Hellström M, Scheidl SJ, Abramsson A, Renner O, Lindahl P, Cho H, Kehrl J and Betsholtz C: Transcription profiling of platelet-derived growth factor-B-deficient mouse embryos identifies RGS5 as a novel marker for pericytes and vascular smooth muscle cells. Am J Pathol 162: 721-729, 2003.

5. Cho H, Kozasa T, Bondjers C, Betsholtz C and Kehrl JH: Pericyte-specific expression of Rgs5: Implications for PDGF and EDG receptor signaling during vascular maturation. FASEB J 17: 440-442, 2003.

6. Seki N, Sugano S, Suzuki Y, Nakagawara A, Ohira M, Muramatsu M, Saito T and Hori T: Isolation, tissue expression, and chromosomal assignment of human $R G S 5$, a novel G-protein signaling regulator gene. J Hum Genet 43: 202-205, 1998.

7. Li H, He C, Feng J, Zhang Y, Tang Q, Bian Z, Bai X, Zhou H, Jiang H, Heximer SP, et al: Regulator of $G$ protein signaling 5 protects against cardiac hypertrophy and fibrosis during biomechanical stress of pressure overload. Proc Natl Acad Sci USA 107: 13818-13823, 2010.

8. Cho H, Park C, Hwang IY, Han SB, Schimel D, Despres D and Kehrl JH: Rgs 5 targeting leads to chronic low blood pressure and a lean body habitus. Mol Cell Biol 28: 2590-2597, 2008.

9. Li J, Adams LD, Wang X, Pabon L, Schwartz SM, Sane DC and Geary RL: Regulator of G protein signaling 5 marks peripheral arterial smooth muscle cells and is downregulated in atherosclerotic plaque. J Vasc Surg 40: 519-528, 2004.

10. Takata Y, Liu J, Yin F, Collins AR, Lyon CJ, Lee CH, Atkins AR, Downes M, Barish GD, Evans RM, et al: PPARdelta-mediated antiinflammatory mechanisms inhibit angiotensin II-accelerated atherosclerosis. Proc Natl Acad Sci USA 105: 4277-4282, 2008.

11. Deng W, Wang X, Xiao J, Chen K, Zhou H, Shen D, Li H and Tang Q: Loss of regulator of $\mathrm{G}$ protein signaling 5 exacerbates obesity, hepatic steatosis, inflammation and insulin resistance. PLoS One 7: e30256, 2012.

12. Yang Z, Balenga N, Cooper PR, Damera G, Edwards R, Brightling CE, Panettieri RA Jr and Druey KM: Regulator of G-protein signaling-5 inhibits bronchial smooth muscle contraction in severe asthma. Am J Respir Cell Mol Biol 46: 823-832, 2012.

13. Bell SE, Mavila A, Salazar R, Bayless KJ, Kanagala S, Maxwell SA and Davis GE: Differential gene expression during capillary morphogenesis in 3D collagen matrices: Regulated expression of genes involved in basement membrane matrix assembly, cell cycle progression, cellular differentiation and G-protein signaling. J Cell Sci 114: 2755-2773, 2001.

14. Berger M, Bergers G, Arnold B, Hämmerling GJ and Ganss R: Regulator of G-protein signaling-5 induction in pericytes coincides with active vessel remodeling during neovascularization. Blood 105: 1094-1101, 2005.

15. Arnold C, Feldner A, Pfisterer L, Hödebeck M, Troidl K, Genové G, Wieland T, Hecker M and Korff T: RGS5 promotes arterial growth during arteriogenesis. EMBO Mol Med 6: 1075-1089, 2014.

16. Furuya M, Nishiyama M, Kimura S, Suyama T, Naya Y, Ito H, Nikaido $T$ and Ishikura $\mathrm{H}$ : Expression of regulator of $\mathrm{G}$ protein signalling protein 5 (RGS5) in the tumour vasculature of human renal cell carcinoma. J Pathol 203: 551-558, 2004.

17. Chen X, Higgins J, Cheung ST, Li R, Mason V, Montgomery K, Fan ST, van de Rijn M and So S: Novel endothelial cell markers in hepatocellular carcinoma. Mod Pathol 17: 1198-1210, 2004.

18. Silini A, Ghilardi C, Figini S, Sangalli F, Fruscio R, Dahse R, Pedley RB, Giavazzi R and Bani M: Regulator of G-protein signaling 5 (RGS5) protein: A novel marker of cancer vasculature elicited and sustained by the tumor's proangiogenic microenvironment. Cell Mol Life Sci 69: 1167-1178, 2012.

19. Wang JH, Huang WS, Hu CR, Guan XX, Zhou HB and Chen LB: Relationship between RGS5 expression and differentiation and angiogenesis of gastric carcinoma. World $\mathrm{J}$ Gastroenterol 16: 5642-5646, 2010.

20. Koh J, Dar M, Untch BR, Dixit D, Shi Y, Yang Z, Adam MA, Dressman H, Wang X, Gesty-Palmer D, et al: Regulator of G protein signaling 5 is highly expressed in parathyroid tumors and inhibits signaling by the calcium-sensing receptor. Mol Endocrinol 25: 867-876, 2011.

21. Huang G, Song H, Wang R, Han X and Chen L: The relationship between RGS5 expression and cancer differentiation and metastasis in non-small cell lung cancer. J Surg Oncol 105: 420-424, 2012. 
22. Yang X, Cai W, Xu Z, Chen J, Li C, Liu S, Yang Z, Pan Q, Li M, Ma J, et al: High efficacy and minimal peptide required for the anti-angiogenic and anti-hepatocarcinoma activities of plasminogen K5. J Cell Mol Med 14: 2519-2530, 2010.

23. Xu Z, Fang S, Zuo Y, Zhang Y, Cheng R, Wang Q, Yang Z, Cai W, Ma J, Yang X, et al: Combination of pigment epitheliumderived factor with radiotherapy enhances the antitumor effects on nasopharyngeal carcinoma by downregulating vascular endothelial growth factor expression and angiogenesis. Cancer Sci 102: 1789-1798, 2011.

24. Fei $\mathrm{P}$ and El-Deiry WS: P53 and radiation responses. Oncogene 22: 5774-5783, 2003.

25. Venkatakrishnan AJ, Deupi X, Lebon G, Tate CG, Schertler GF and Babu MM: Molecular signatures of G-protein-coupled receptors. Nature 494: 185-194, 2013.

26. Karnik SS, Gogonea C, Patil S, Saad Y and Takezako T: Activation of G-protein-coupled receptors: A common molecular mechanism. Trends Endocrinol Metab 14: 431-437, 2003.

27. O'Callaghan K, Kuliopulos A and Covic L: Turning receptors on and off with intracellular pepducins: New insights into G-protein-coupled receptor drug development. J Biol Chem 287: 12787-12796, 2012.

28. Roman DL and Traynor JR: Regulators of G protein signaling (RGS) proteins as drug targets: Modulating G-protein-coupled receptor (GPCR) signal transduction. J Med Chem 54: 7433-7440, 2011.

29. Dorsam RT and Gutkind JS: G-protein-coupled receptors and cancer. Nat Rev Cancer 7: 79-94, 2007.

30. Nishiura H, Nonaka H, Revollo IS, Semba U, Li Y, Ota Y, Irie A, Harada K, Kehrl JH and Yamamoto T: Pro- and anti-apoptotic dual functions of the C5a receptor: Involvement of regulator of $\mathrm{G}$ protein signaling 3 and extracellular signal-regulated kinase. Lab Invest 89: 676-694, 2009.

31. Liang G, Bansal G, Xie Z and Druey KM: RGS16 inhibits breast cancer cell growth by mitigating phosphatidylinositol 3-kinase signaling. J Biol Chem 284: 21719-21727, 2009.

32. Altman MK, Nguyen DT, Patel SB, Fambrough JM, Beedle AM, Hardman WJ and Murph MM: Regulator of G-protein signaling 5 reduces HeyA8 ovarian cancer cell proliferation and extends survival in a murine tumor model. Biochem Res Int 2012: 518437 , 2012.
33. Maity B, Yang J, Huang J, Askeland RW, Bera S and Fisher RA: Regulator of G protein signaling 6 (RGS6) induces apoptosis via a mitochondrial-dependent pathway not involving its GTPaseactivating protein activity. J Biol Chem 286: 1409-1419, 2011

34. Jin Y, An X, Ye Z, Cully B, Wu J and Li J: RGS5, a hypoxia-inducible apoptotic stimulator in endothelial cells. J Biol Chem 284 23436-23443, 2009.

35. Czabotar PE, Lessene G, Strasser A and Adams JM: Control of apoptosis by the BCL-2 protein family: Implications for physiology and therapy. Nat Rev Mol Cell Biol 15: 49-63, 2014.

36. Mu XM, Shi W, Sun LX, Li H, Wang YR, Jiang ZZ and Zhang LY: Pristimerin inhibits breast cancer cell migration by up-regulating regulator of $\mathrm{G}$ protein signaling 4 expression. Asian Pac J Cancer Prev 13: 1097-1104, 2012.

37. Xie Y, Wolff DW, Wei T, Wang B, Deng C, Kirui JK, Jiang H, Qin J, Abel PW and Tu Y: Breast cancer migration and invasion depend on proteasome degradation of regulator of G-protein signaling 4. Cancer Res 69: 5743-5751, 2009.

38. Schofield AV and Bernard O: Rho-associated coiled-coil kinase (ROCK) signaling and disease. Crit Rev Biochem Mol Biol 48: 301-316, 2013.

39. Rane CK and Minden A: P21 activated kinases: Structure, regulation, and functions. Small GTPases 5: 5, 2014.

40. Luanpitpong S, Talbott SJ, Rojanasakul Y, Nimmannit U, Pongrakhananon V, Wang L and Chanvorachote P: Regulation of lung cancer cell migration and invasion by reactive oxygen species and caveolin-1. J Biol Chem 285: 38832-38840, 2010.

41. Hooks SB, Callihan P, Altman MK, Hurst JH, Ali MW and Murph MM: Regulators of G-Protein signaling RGS10 and RGS17 regulate chemoresistance in ovarian cancer cells. Mol Cancer 9: 289, 2010.

42. Huang J, Yang J, Maity B, Mayuzumi D and Fisher RA: Regulator of $\mathrm{G}$ protein signaling 6 mediates doxorubicin-induced ATM and p53 activation by a reactive oxygen species-dependent mechanism. Cancer Res 71: 6310-6319, 2011. 